\title{
The Utility of a High-intensity Exercise Protocol to Prospectively Assess ACL Injury Risk
}

Authors

Affiliations
F. M. Bossuyt' ${ }^{1}$, F. García-Pinillos ${ }^{2}$, R. M. F. Raja Azidin' ${ }^{1}$, J. Vanrenterghem ${ }^{3}$, M. A. Robinson ${ }^{4}$

Research Institute of Sport and Exercise Sciences, Liverpool John Moores University, Liverpool, United Kingdom Department of Corporal Expression, University of Jaen, Jaen, Spain

Research Institute for Sport and Exercise, Liverpool John Moores University, Liverpool, United Kingdom

${ }^{4}$ Sport Science, Liverpool John Moores University, Liverpool, United Kingdom
Key words

- biomechanics

anterior cruciate ligament

isokinetic dynamometry accepted after revision June 25, 2015

Bibliography DOI http://dx.doi.org/ 10.1055/s-0035-1555930 Published online: October 28, 2015 Int J Sports Med 2016; 37 : 125-133 (c) Georg Thieme Verlag KG Stuttgart · New York ISSN 0172-4622

\section{Correspondence}

Fransiska Marie Bossuyt, Msc Research Institute of Sport and Exercise Sciences

Liverpool John Moores University

Tom Reilly Building

Liverpool

United Kingdom

L3 3AF

Tel.: + 44/151/904 6259

Fax: + 44/151/904 6259

fransiskabossuyt@gmail.com

\section{Abstract}

$\nabla$

This study investigated the utility of a 5-min high-intensity exercise protocol $\left(\mathrm{SAFT}^{5}\right)$ to include in prospective cohort studies investigating ACL injury risk. 15 active females were tested on 2 occasions during which their non-dominant leg was analysed before SAFT $^{5}$ (PRE), immediately after (POST0), $15 \mathrm{~min}$ after (POST15), and $30 \mathrm{~min}$ after (POST30). On the first occasion, testing included 5 maximum isokinetic contractions for eccentric and concentric hamstring and concentric quadriceps and on the second occasion, 3 trials of 2 landing tasks (i.e., single-leg hop and drop vertical jump) were conducted. Results

\section{Introduction}

\section{$\nabla$}

Risk factors of ACL injuries can only be defined with the highest level of evidence when prospectively assessed [39]. Muscular and biomechanical ACL injury risk factors have been studied extensively as they are modifiable through training [41]. Muscular risk factors include reduced eccentric hamstring peak torque $\left(\mathrm{H}_{\mathrm{ecc}}\right)$ and reduced hamstring/quadriceps ratio $(\mathrm{H} / \mathrm{Q})$ [45]. Reduced hamstring strength is believed to permit increased anterior tibial translation and in turn increase ACL strain [2]. Biomechanical risk factors include increased peak knee abduction moment and peak vertical ground reaction force $\left(\mathrm{VGRF}_{\text {peak }}\right)$ [21]. Reduced knee flexion at initial contact (IC) during landing has been associated with increased anterior tibial translation $[6,7]$, which causes increased ACL loading $[6,8,9]$. Additionally, increased knee internal rotation has been associated with increased ACL loading $[9,10]$. It is important to note that fatigue (i.e., loss of maximum or potential performance) alters these factors which may further increase injury risk $[11,12]$. Additionally, evidence shows showed a reduced eccentric hamstring peak torque at POST0, POST15 and POST30 (p<0.05) and a reduced functional $\mathrm{HQ}$ ratio (Hecc/Qcon) at POST15 and POST30 $(p<0.05)$. Additionally, a more extended knee angle at POST30 $(p<0.05)$ and increased knee internal rotation angle at POST0 and POST15 $(p<0.05)$ were found in a single-leg hop. SAFT ${ }^{5}$ altered landing strategies associated with increased ACL injury risk and similar to observations from match simulations. Our findings therefore support the utility of a high-intensity exercise protocol such as $\mathrm{SAFT}^{5}$ to strengthen injury screening tests and to include in prospective cohort studies where time constraints apply. that most injuries occur at the end of a soccer match $[12,13]$. These findings suggest that fatigue plays a crucial role on muscular and biomechanical risk factors in the mechanism of ACL injury [19]. To date, however, no prospective studies have included exercise-induced fatigue in their screening protocols, most likely due to the conflict between screening a large cohort and the time-consuming nature of inducing fatigue.

Fatigue has been induced in a number of ways, some of which are more related to dynamic activities than others. Previous research has suggested, however, that including functional movements as part of the protocol is key to revealing specific match-play-induced deficits which can increase ACL injury risk $[14,15]$. As such the effects of a shuttle run revealed changes in transverse plane kinematics in sidestep cutting as increased external rotation of hip, knee and ankle angle at IC and increased knee internal rotation angle during stance [40]. In another study, a treadmill-based soccer match simulation reduced $\mathrm{H} / \mathrm{Q}[16]$. Finally, a soccer-specific match simulation $\left(\mathrm{SAFT}^{90}\right)$ which included multidirectional movements, high accelerations and decel- 
erations, and which was shown to be a valid simulation of match play [26], caused significant reductions in $\mathrm{H}_{\text {ecc }}$ and in $\mathrm{H} / \mathrm{Q}$ $[36,43,44]$. Fatigue induced by soccer match play $(90 \mathrm{~min})$ has been suggested to be a combination of both central (altered motor commands from the motor cortex) and peripheral fatigue (metabolite accumulation, limitations in energy supply, reduced blood flow and neuromuscular mechanisms) [37,50]. Additionally it has been suggested that during soccer, players experience fatigue in several different ways: (1) disturbed muscle ion homeostasis during temporary fatigue after short bursts of highintensity exercises, (2) lowered muscle temperature (e.g., at the beginning of the second half) and (3) through muscle glycogen and dehydration as experienced towards the end of a game [32]. Whilst full-length match simulations would be considered most ideal for simulating the effects of match play, the development and evaluation of short-term protocols is needed to include match-play-induced fatigue assessment within prospective studies. It is important to acknowledge the influence of intensity, duration and type of contraction on the mechanisms of fatigue [3]. As far as we are aware however, no previous study has directly compared neuromuscular responses between shortterm protocols and full-length match simulations. Nevertheless, previous findings [27] found similar biomechanical alterations in response to a short-duration high-intensity protocol and a longer duration protocol $(30 \mathrm{~min}) .2$ studies involving shortterm protocols, the first including vertical jumps followed by 30-m sprint, and the second including series of athletic exercises (countermovement jump (CMJ), step up/down, squat and shuttle run), resulted in decreased knee flexion angles at IC and increased knee abduction moments in sidestep cutting [10] and stop-jump tasks [8]. Finally, a short-term protocol based on continuous drills (step up/down and plyometric bounding) caused increased knee abduction angles/moments and increased knee internal rotation angles in a drop vertical jump (DVJ) [31]. It is important to note that all these protocols induced fatigue until maximum exhaustion which is not representative of match play, and inappropriate for the inclusion in prospective studies as this implies differences in duration or amount of repetitions.

Few if any studies have focused on short-term protocols that simulate match play of dynamic sports (i.e., sport which involves high accelerations and decelerations and typically involves interactions with an object (ball, racket, etc.)). Therefore, the aim of this study was to investigate how a short-term high-intensity exercise protocol $\left(\mathrm{SAFT}^{5}\right)$, based on a long-duration match-play simulation $\left(\mathrm{SAFT}^{90}\right)$ [26], affects muscular and biomechanical markers of ACL injury in recreationally active females. It was hypothesized that $\mathrm{SAFT}^{5}$ would result in changes in markers of ACL injury risk.

\section{Methods \\ $\nabla$}

\section{Participants}

15 females (age: $22 \pm 3$ years, height: $1.68 \pm 0.07 \mathrm{~m}$, mass: $70.8 \pm 8.9 \mathrm{~kg}$ ) were tested following a sample-size estimation from a functional fatigue protocol to observe a $5^{\circ}$ difference in knee flexion angle at $80 \%$ statistical power and alpha $=0.05$ [11,31]. All participants met the inclusion criteria: (1) female, (2) recreationally active (i.e., 3 sessions per week) in dynamic sports (hockey, netball, etc.), (3) did not suffer from an ACL injury and (4) did not suffer from any other lower limb injury within the last 6 months before data collection. Ethical approval was granted by the university ethics committee and all participants provided informed consent according to IJSM ethical standards [18].

\section{Design}

This was a cross-sectional study with a repeated measures design. Initially participants were familiarized with the protocol and assessment methods, height and weight were recorded, and maximum jump height (JumpHeight ${ }_{\max }$ ) was defined. This was followed by 2 testing sessions which were separated by at least 3 days. Both sessions included a dynamic warm-up, the SAFT ${ }^{5}$ protocol, a pre-test (PRE), a test immediately following $\mathrm{SAFT}^{5}$ (POST0), after $15 \mathrm{~min}$ of passive rest (POST15) and after $30 \mathrm{~min}$ of passive rest (POST30). These moments in time were selected in order to define the prolonged effect of SAFT ${ }^{5}$. This information is required for the inclusion of SAFT ${ }^{5}$ in a prospective study protocol which could consist of measurements before and after $\mathrm{SAFT}^{5}$. In addition, this information would be useful for the design of activity-rest cycles to minimize the potential for fatigue-related injury. Different parameters were assessed during each session and between participants the order of sessions was randomly assigned. Participants were instructed to avoid strenuous exercises $48 \mathrm{~h}$ prior to testing.

\section{Exercise protocol}

SAFT $^{5}$ is based on the first 5 min of SAFT ${ }^{90}[26,36]$. The distance of the SAFT ${ }^{90}$ protocol was modified to $15 \mathrm{~m}$ in order to make the SAFT $^{5}$ course feasible in our laboratory ( $\bullet$ Fig. 1 and $\odot$ Table 1 ). The intensity of SAFT ${ }^{5}$ was increased by adding high-intensity exercises, based on previous studies $[11,31,51]$ and pilot work $[9,54]$. Pilot work aimed at defining the activity profile of the protocol by investigating the implementation of functional highintensity movements. Several variations of the protocol based on different high-intensity exercises, amount of repetitions and order of exercises were explored by monitoring HR and RPE during the protocol which represented the intensity. The final protocol was selected in accordance with the following criteria: (1)

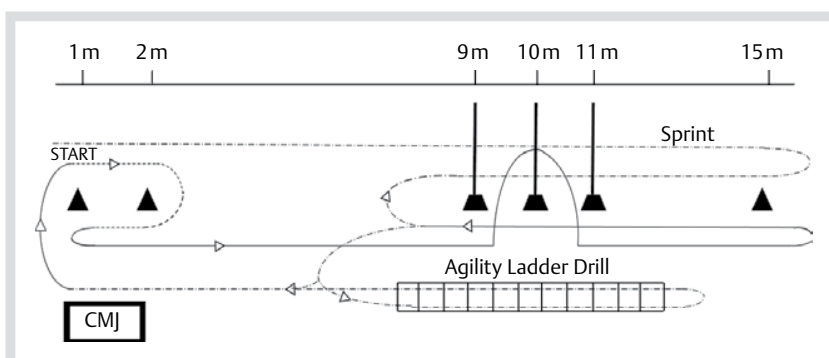

Fig. 1 The SAFT ${ }^{5}$ course. The participant received anticipatory instructions about the task and the speed for $5 \mathrm{~min}$. The dotted line at the start of the course represents either upwards and backwards ("up jog") or sideways running ("side") around the second cone. The solid line represents forward running and sidestep cutting around the middle pole. The speed of the task was either "jog" or "stride". Stride represented a speed between a jog and sprint. Once the participant arrived back at the first pole a second instruction was given. This instruction was either: (1) "sprint" or "jog", which meant that the participant jogged or sprinted; (2) "agility ladder", which meant that the participant performed the agility drill of running forwards with one foot per square and performing a final sprint once finished with the agility ladder drill; (3) "CMJ" or "scissors", which meant that the participant performed $10 \mathrm{CM}$ )s ( 1 maximum jump on a jump mat and 9 jumps at $80 \%$ of JumpHeight $t_{\max }$ ) or 10 scissors at the black square that is represented on the fig. 
HR and RPE presented a similar overall pattern as during SAFT ${ }^{90}$ and actual game play [24,36], and (2) practical and personal observations of the researchers. As such it was decided to include the following 3 exercises: a CMJ at $80 \%$ of their JumpHeight max $_{\text {, }}$, an agility ladder drill (one foot per square) and a 'jump scissors' task (jumping from unilateral lunge with left leg forward and hands placed on the hips, to unilateral lunge with right leg forward) ( $\bullet$ Table 1$)$. During the protocol, white tape was placed onto a wall which represented $80 \%$ JumpHeight $_{\max }$, participants had to touch the white tape during every jump and received verbal feedback if they didn't reach the tape.

Table 1 Activity profile of SAFT ${ }^{5}$ compared with the activity profile of 5 min of $\mathrm{SAFT}^{90}$ with the order, speed and duration of each task represented.

\begin{tabular}{|c|c|c|c|c|}
\hline No & Activity SAFT ${ }^{5}$ & Activity SAFT ${ }^{90}$ & Speed $(\mathrm{m} / \mathrm{s})$ & Time (s) \\
\hline 0 & $10 \times$ scissors & & & \\
\hline 1 & stand & stand & 0 & 4 \\
\hline 2 & jog & jog & 2.86 & 10 \\
\hline 3 & $\begin{array}{l}\text { CMJ }(1 \times C M]_{\max }+9 \times 80 \% \\
\left.\text { JumpHeight } t_{\max }\right)\end{array}$ & walk & 1.39 & 17 \\
\hline 4 & stride & stride & 4.17 & 7 \\
\hline 5 & agility ladder drill & walk & 1.39 & 17 \\
\hline 6 & jog & jog & 2.86 & 10 \\
\hline 7 & sprint & sprint & 5.58 & 6 \\
\hline 8 & stand & stand & 0 & 4 \\
\hline 9 & jog & jog & 2.86 & 10 \\
\hline 10 & $10 \times$ Scissors & walk & 1.39 & 17 \\
\hline 11 & jog & jog & 2.86 & 10 \\
\hline 12 & $\begin{array}{l}\text { CMJ }\left(1 \times C M J_{\max }+9 \times 80 \%\right. \\
\left.\text { JumpHeight } t_{\max }\right)\end{array}$ & walk & 1.39 & 17 \\
\hline 13 & stride & stride & 4.17 & 7 \\
\hline 14 & agility ladder drill & walk & 1.39 & 17 \\
\hline 15 & stand & stand & 0 & 4 \\
\hline 16 & jog & jog & 2.86 & 10 \\
\hline 17 & $10 \times$ Scissors & walk & 1.39 & 17 \\
\hline 18 & jog & jog & 2.86 & 10 \\
\hline 19 & jog & jog & 2.86 & 10 \\
\hline 20 & jog & jog & 2.86 & 10 \\
\hline 21 & $\begin{array}{l}\text { CMJ }(1 \times C M]_{\max }+9 \times 80 \% \\
\left.\text { JumpHeight } t_{\max }\right)\end{array}$ & walk & 1.39 & 17 \\
\hline 22 & jog & jog & 2.86 & 10 \\
\hline 23 & agility ladder drill & walk & 1.39 & 17 \\
\hline 24 & stride & stride & 4.17 & 7 \\
\hline 25 & stand & stand & 0 & 4 \\
\hline 26 & $10 \times$ scissors & walk & 1.39 & 17 \\
\hline 27 & jog & jog & 2.86 & 10 \\
\hline 28 & sprint & walk & 1.39 & 17 \\
\hline
\end{tabular}

\section{Data collection}

At one test session maximum voluntary contractions (MVCs) of concentric hamstring strength $\left(\mathrm{H}_{\text {con }}\right)$, concentric quadriceps strength $\left(\mathrm{Q}_{\mathrm{con}}\right)$ and $\mathrm{H}_{\mathrm{ecc}}$ were assessed by an isokinetic dynamometer (IKD, Biodex System 3, Shirley, NY). The nondominant leg (i.e., non-preferred leg to kick a ball with) was tested as it has been identified as the most vulnerable to ACL injury in females $[6,38]$. Concentric MVCs consisted of repeated knee flexion and extension contractions within $90^{\circ}$ RoM at $120^{\circ} \mathrm{s}$. During eccentric MVCs, participants resisted against the passive external knee extension moment of the IKD over $90^{\circ}$ RoM and at $120^{\circ} / \mathrm{s}[35,43]$. Participants were verbally encouraged and 5 repetitions were measured for each task with one min rest between different contractions. The order of assessment was randomly assigned.

At the other test session, participants wore tight-fitting clothes and measurements consisted of 3D motion and force analysis of DVJ and single-leg hop (SLH). For the DVJ, participants were instructed to drop off a $30-\mathrm{cm}$ high box (feet $20 \mathrm{~cm}$ apart), and land with each foot on separate force platforms, immediately rebounding for a maximum vertical jump. For the SLH, participants were instructed to stand on the non-dominant leg and hop forward to cover a distance of $75 \%$ of body height [33] in order to use a standardised distance adjusted to personal dimensions. 3 successful trials of each task were recorded with trials excluded if the participant lost balance less than $2 \mathrm{~s}$ after landing.

10 optoelectronic cameras sampling at $250 \mathrm{~Hz}$ (OQUS 3, Qualysis $A B$, Gothenburg, Sweden) were used to collect 3D motion data. Spherical reflective markers were attached to lower limb and trunk according to the LJMU kinematic model [49], which has established reliability [28]. One static and 4 functional motion trials were recorded to define functional hip and knee joint axes, after which anatomy-defining markers were removed. GRF were collected simultaneously from 2 force platforms at $1500 \mathrm{~Hz}$ (Kistler, Winterthur, Switzerland).

Additional measurements for both sessions, recorded every 5 min throughout the sessions and during $\mathrm{SAFT}^{5}$, included JumpHeight $_{\max }$ with a jump mat (Probotics, Inc., Huntsville, AL) in order to assess fatigue as a reduction of performance, heart rate (HR) (Polar heart rate system, Electro, Finland) and rate of perceived exertion (RPE) (20-point Borg scale). Timing and order of each measurement is represented in $\odot$ Table 2.

\section{Data analysis}

Kinematic and kinetic data were calculated within Visual 3D (C-Motion, Germantown, MD). Marker trajectories and forces

Table 2 Timing and order of measurements (one maximum counter movement jump (CMJ), heart rate (HR), rate of perceived exertion (RPE), kinematics and kinetics of a single leg hop (SLH) and drop vertical jump (DVJ) of the non-dominant leg and maximum voluntary contractions of concentric and eccentric hamstring strength and concentric quadriceps (IKD)) during the 2 testing sessions.

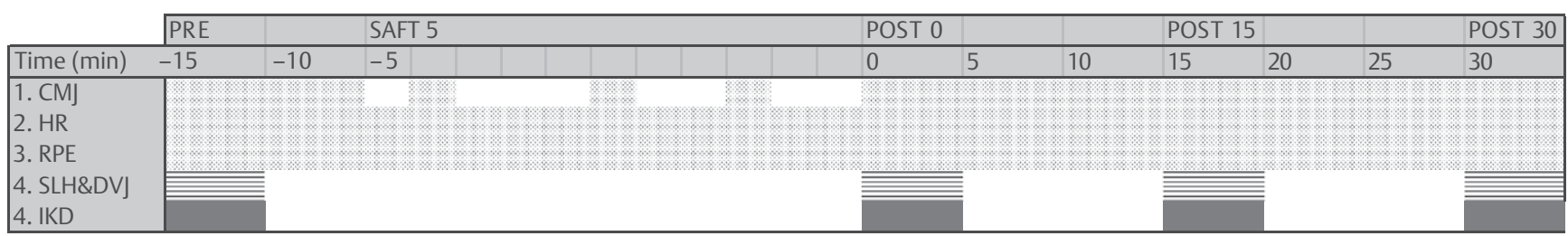

\begin{tabular}{|ll|}
\hline Test $1 \& 2$ & \\
Test 1 & \\
Test 2 & \\
\hline \hline
\end{tabular}




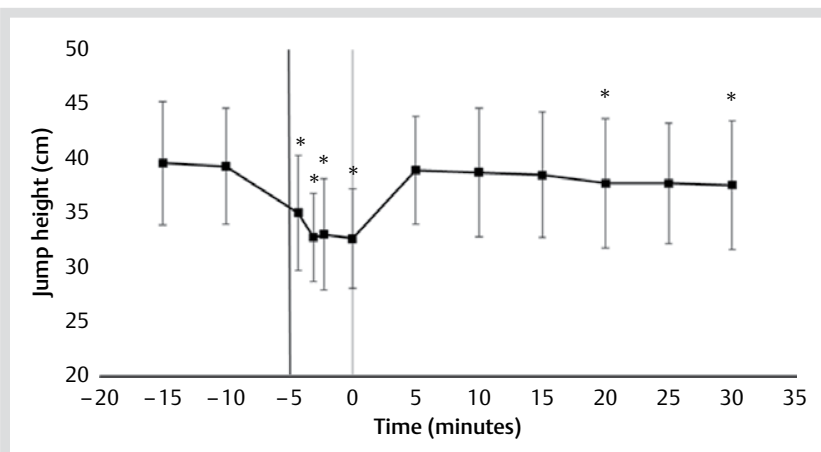

Fig. 2 Maximum jump height pre ( $-10 \mathrm{~min}$ to $0 \mathrm{~min}$ ), during ( $0 \mathrm{~min}$ to $5 \mathrm{~min}$ ) and post ( $5 \mathrm{~min}$ to $35 \mathrm{~min}$ ) SAFT ${ }^{5}$. Vertical lines represent beginning (time -5 ) and end (time 0 ) of $\mathrm{SAFT}^{5}$. denotes statistically significant difference $(p<0.05)$.

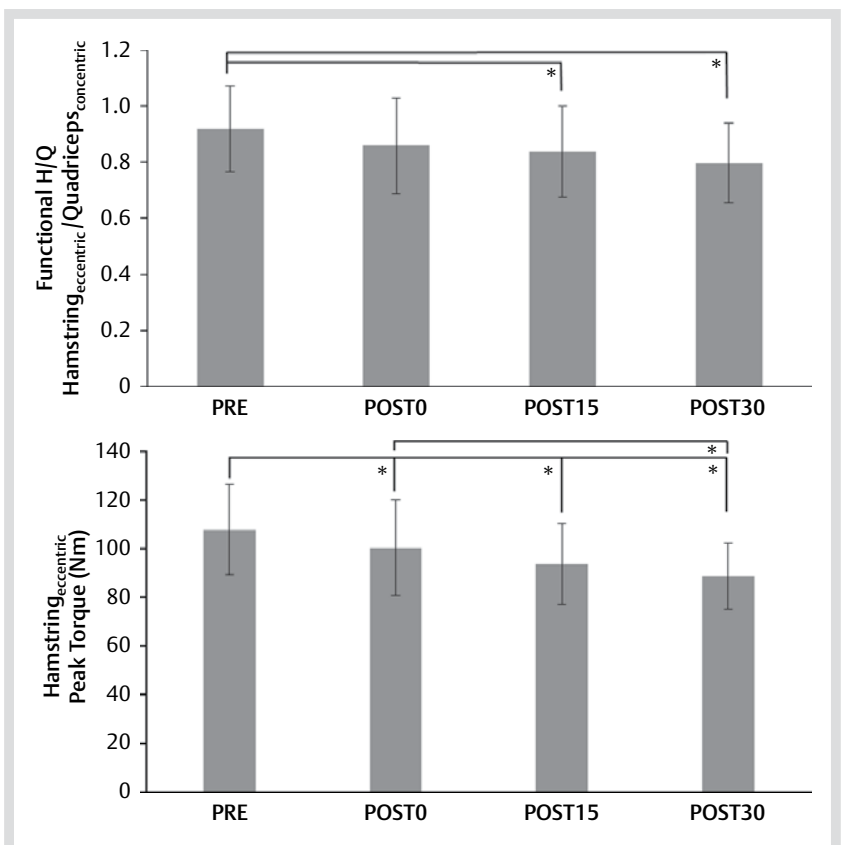

Fig. 3 Functional $\mathrm{HQ}$ ratio $\left(\mathrm{H}_{\text {eccentric }} / \mathrm{Q}_{\text {concentric }}\right)$ and hamstring eccentric peak torque pre, during and post ( $5 \mathrm{~min}, 20 \mathrm{~min}$ and $35 \mathrm{~min}$ ) SAFT ${ }^{5}$. ${ }^{*}$ denotes statistically significant difference $(p<0.05)$.

were filtered through a Butterworth and a critically damped low-pass filter with $20-\mathrm{Hz}$ cut-off frequencies and normalized to 101 time nodes. IC and take-off were defined as the instant when GRF exceeded or reached below $10 \mathrm{~N}$ [10]. The stance phase of an SLH was defined as one second after IC. Only the first landing of the DVJ was used for analysis [1]. Euler rotations (X-Y$Z$ ) were used for joint angle calculations and knee moments were obtained by inverse dynamics [31] and are reported as external moments. IKD data were gravity-corrected and analysed with a custom Matlab (MathWorks, Inc., Natick, MA, USA) program. Peak torques were calculated from a polynomial fit of data points that met the criteria within a $10 \%$ tolerance (velocity: $120^{\circ} / \mathrm{s}$, RoM at least $70^{\circ}$ for $\mathrm{H}_{\text {con }}$ and $\mathrm{Q}_{\text {con }}$, and RoM at least $50^{\circ}$ for $\left.\mathrm{H}_{\mathrm{ecc}}\right)$. Finally, the functional HQ ratio $\left(\mathrm{H}_{\mathrm{ecc}} / \mathrm{Q}_{\mathrm{con}}\right)$ which was previously presented as a valid representation of muscle-specific exertion induced by football match play [12] and conventional
HQ ratio $\left(\mathrm{H}_{\text {con }} / \mathrm{Q}_{\text {con }}\right)$ were calculated. Dependent variables of interest were JumpHeight max $_{\text {m }}, \mathrm{HR}, \mathrm{RPE}, \mathrm{H}_{\text {ecc }} / \mathrm{Q}_{\text {con }}, \mathrm{H}_{\text {con }} / \mathrm{Q}_{\text {con }}, \mathrm{H}_{\text {con }}$, $\mathrm{H}_{\text {ecc }}$ and $\mathrm{Q}_{\mathrm{con}}$, knee flexion angle and transverse plane knee angle at IC, peak knee abduction moment and VGRF peak.

\section{Statistical treatment}

Data are presented as means \pm standard deviation. One-way repeated measures ANOVAs were used to assess meaningful variations across time for each variable. Percentage differences of jump height, $\mathrm{H}_{\mathrm{ecc}}, \mathrm{H}_{\text {con }}, \mathrm{Q}_{\text {con }}, \mathrm{H}_{\mathrm{ecc}} / \mathrm{Q}_{\text {con }}$ and $\mathrm{H}_{\text {con }} / \mathrm{Q}_{\text {con }}$ were calculated and $\mathrm{HR}$ was presented as a percentage of the estimated maximum HR (HRmax $=220$-age). If statistical significance was found, pairwise comparisons were applied with Bonferroni corrections to reduce the risk of type- 1 errors. Reliability of the 2 testing sessions was confirmed by (1) low typical error and limits of agreement for RPE (Pre: $1,[-2 ;-1]$; During: $1[-1 ; 1]$; Post: $1[-1 ; 0])$ and HR (Pre: 5 beats/min $[-5 ; 3]$; During: 2 beats/min $[1,4]$; Post: 3 beats/min $[-5 ;-1])$ and $(2)$ uniform errors based on observed homoscedasticity. All statistical analyses were performed in SPSS (Version 21.0, Chicago, IL) $(\alpha=0.05)$.

\section{Results}

$\nabla$

There was a significant effect of $\mathrm{SAFT}^{5}$ on $\mathrm{HR}\left(F_{3.99,47.88}=257.78\right.$, $p<0.001)$ and $\operatorname{RPE}\left(F_{3.66,43.90}=140.48, p<0.001\right)$ as these variables significantly increased up to $89 \pm 4 \% \mathrm{HR}_{\max }$ and $17 \mathrm{RPE}$ during $\operatorname{SAFT}^{5}(p<0.001)$ and up to $60 \% \mathrm{HR}_{\max }$ and $11 \mathrm{RPE} 5 \mathrm{~min}$ post-SAFT ${ }^{5}(p<0.001)$.

JumpHeight $_{\max }$ significantly reduced $\left(F_{3.41,47.80}=39.33, p<0.001\right)$ (๑ Fig. 2) with a reduction during $\mathrm{SAFT}^{5}$ by $5.8 \pm 2.0 \mathrm{~cm}(15 \%$, $p<0.05)$ and after 20 and 30 min passive rest by $1.9 \pm 0.1 \mathrm{~cm} \mathrm{(5 \% )}$ $(p=0.019$ and $p=0.011)$.

A significant main effect of $\mathrm{SAFT}^{5}$ on $\mathrm{H}_{\text {ecc }}$ was found $\left(F_{2.27}\right.$, $38.29=11.01, p<0.001$ ) ( $\odot$ Fig. 3 ) with a significant reduction at POST0 (7\%, $p=0.029)$, POST15 (13\%, $p<0.001)$ and POST30 $(18 \%$, $p<0.001)$ compared to PRE, and at POST30 compared to POST0 $\left(12 \%, p=0.024\right.$,). This caused a significant main effect in the $\mathrm{H}_{\mathrm{ecd}}$ $\mathrm{Q}_{\text {con }}\left(F_{2.01,28.14}=4.27, p=0.024\right)$. There was no significant reduction in the $\mathrm{H}_{\mathrm{ecc}} / \mathrm{Q}_{\mathrm{con}}$ immediately post-SAFT ${ }^{5}$, however, POST15 and POST30 showed a significant reduction of $9 \%(p=0.010)$ and $13 \%(p=0.003)$.

There was no significant effect of $\mathrm{SAFT}^{5}$ on $\mathrm{H}_{\text {con }} / \mathrm{Q}_{\text {con }}$ $\left(F_{2.93,41.01}=0.63, p=0.566\right)$. Even though there was a significant effect of SAFT ${ }^{5}$ on both $\mathrm{H}_{\text {con }}\left(F_{2.82,39.42}=3.91, p=0.025\right)$ and $\mathrm{Q}_{\text {con }}$ $\left(F_{1.83,25.66}=3.70, p=0.042\right)$ there was only a significant reduction between PRE and POST30 in $\mathrm{H}_{\text {con }}(7 \%, p=0.014)$ and $\mathrm{Q}_{\text {con }}(5 \%$, $p=0.024)$ and between POST0 and POST30 in $\mathrm{H}_{\text {con }}(6 \%, p=0.013)$ and $\mathrm{Q}_{\mathrm{con}}(4 \%, p=0.045)$.

Results of the SLH indicated that SAFT ${ }^{5}$ induced (1) a signifi-

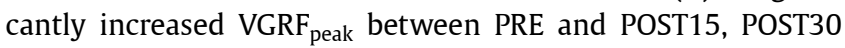
and between POST0 and POST15, POST30, (2) a significantly more extended knee $\left(2.2 \pm 2.6^{\circ}\right)$ between PRE and POST30 and (3) a significantly increased internal rotation angle between PRE and POST0 $\left(4.1 \pm 4.5^{\circ}\right)$ and POST15 $\left(4.5 \pm 3.8^{\circ}\right.$ ) ( Table 3). No significant effect of SAFT $^{5}$ was found on peak knee-abduction moment in SLH and on any of dependent variables in DVJ. Supplementary files include kinematics and kinetics of an SLH (Appendix 1, 2). 


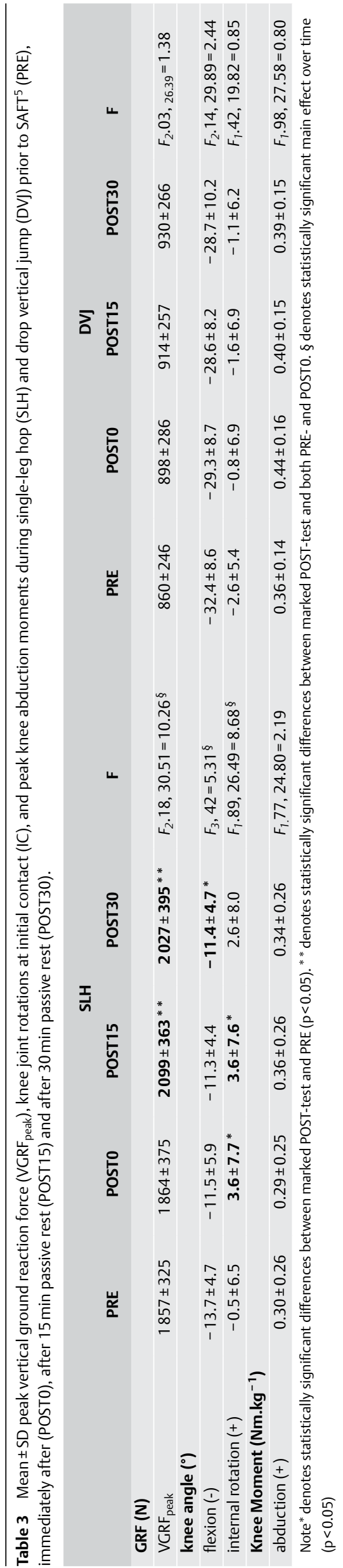

\section{Discussion}

This study determined whether the SAFT ${ }^{5}$, a high-intensity exercise protocol, could be effectively used to induce changes in the muscular and biomechanical characteristics that are typically associated with ACL injury risk. The results partly confirmed the hypothesis. Most notably, there was a significant reduction in $\mathrm{H}_{\mathrm{ecc}}$ and $\mathrm{H}_{\mathrm{ecc}} / \mathrm{Q}_{\mathrm{con}}$, and altered landing strategies in SLH. The protocol caused a $15 \%$ drop in jump performance and RPE was rated between 'hard' and 'very hard' with the average HR $(89 \pm 4 \%$ $\mathrm{HR}_{\max }$ ) similar to that of female football match play (87-97\% $\mathrm{HR}_{\max }$ [24]). The intensity of the current protocol therefore appears to induce physiological responses similar to those in longer duration match-play situations. In general, current findings imply the importance of screening athletes after a highintensity functional exercise protocol such as SAFT ${ }^{5}$.

The significant reduction in $\mathrm{H}_{\mathrm{ecc}}$ immediately after SAFT ${ }^{5}$ and POST15 is in agreement with previous studies investigating the effect of a soccer-specific field test over $90 \mathrm{~min}\left(\mathrm{SAFT}^{90}\right)$ [17,43]. The selective occurrence of fatigue in $\mathrm{H}_{\mathrm{ecc}}$ has been explained previously by the presence of more fatigable type- 2 muscle fibres [14], and the high eccentric requirements of the hamstrings in repetitive sprinting and kicking [53] to counteract the anterior shear forces created by the quadriceps. Despite the significant reduction in $\mathrm{H}_{\text {ecc }}$ between PRE and every post-test, $\mathrm{H}_{\mathrm{ecc}} /$ $\mathrm{Q}_{\text {con }}$ was only significantly reduced POST15 and POST30 $[17,43]$. It should be especially noted that at POST30, $\mathrm{H}_{\mathrm{ecc}} / \mathrm{Q}_{\text {con }}$ fell below the at-risk threshold of 0.71 [52]. The delayed reduction of $\mathrm{H}_{\mathrm{ecc}}$ $\mathrm{Q}_{\text {con }}$ can be explained by the short-term high-intensity characteristics of SAFT ${ }^{5}$. Firstly, the occurrence of post activation potentiation (PAP) (i.e., improved muscular performance in response to conditioning stimulus [22]) could have interfered with the effects immediately following $\mathrm{SAFT}^{5}$, as previous work has found an improvement in peak force within the first $5 \mathrm{~min}$ following exercise [46]. $\mathrm{H}_{\mathrm{ecc}} / \mathrm{Q}_{\mathrm{con}}$ is, however, not increased postSAFT $^{5}$, which assumes that PAP is not the main contributor. Secondly, it could be suggested that there is a delay in peripheral fatigue (i.e., fatigue occurring within the muscle itself) immediately post-SAFT ${ }^{5}$ as a recent study concluded that central fatigue (i.e., alterations in the nervous system) manifests prior to reductions in maximal knee-flexor torque in response to $\mathrm{SAFT}^{90}$ [30]. This finding was explained by the earlier and more pronounced effect of central fatigue on explosive force-producing exercises. The delayed onset of peripheral fatigue is represented in the significant reduction in $\mathrm{H}_{\mathrm{ecc}} / \mathrm{Q}_{\mathrm{con}}$ after 15 min passive rest and is in agreement with previous research $[17,26,43]$. There was no significant reduction in $\mathrm{H}_{\text {con }} / \mathrm{Q}_{\text {con }}$ post-SAFT ${ }^{5}$ which further supports the use of $\mathrm{H}_{\mathrm{ecc}} / \mathrm{Q}_{\text {con }}$ for the evaluation of hamstring function during dynamic activities. Based on $\mathrm{H}_{\mathrm{ecc}}$ and $\mathrm{HQ}$ ratios, it could be assumed that SAFT ${ }^{5}$ induces detrimental effects on muscle performance similar to changes after match-play exertion as $\mathrm{SAFT}^{90}$. Detrimental effects are, however, best observed with a 15 -min delay.

In accordance with the hypothesis, SAFT ${ }^{5}$ altered landing strategies in the non-dominant leg of an SLH. The reduced knee-flexion angle and increased knee internal rotation are in accordance with other studies exploring the effect of a short-term fatigue protocol on sidestep and stop-jump tasks $[8,10,11]$. The significant increase in $\mathrm{VGRF}_{\text {peak }}$ post-SAFT ${ }^{5}$ is in contrast with a previous study investigating the effect of a short-term fatigue protocol [11]. It should be noted that SAFT ${ }^{5}$ aimed to replicate match-play exertion and deviated from the mentioned short-term protocols 
by the lack of maximum exhaustion, which increases the stress applied on the body and may cause greater detrimental effects on landing strategies. Several possible speculations could relate to the significantly reduced knee-flexion angle only after $30 \mathrm{~min}$ passive rest: (1) the suggested relationship between exerciserelated changes in fatigue (e.g., decreased landing forces) and knee laxity (e.g., greater knee-extensor loads and knee shear forces), which is dependent on the baseline knee laxity [42], (2) altered muscular activation patterns in response to fatigue as reduced pre-activation of the hamstrings and gastrocnemius could affect the sagittal and transverse plane differently [15], and (3) post-activation potentiation, as described previously, could play a role [46]. Further studies that take these variables into account will need to be undertaken. Maximum exhaustion may alter landing strategies, which are more related to reduced power generation than to altered stabilization mechanisms. This may explain the different findings of the $\mathrm{VGRF}_{\text {peak }}$. Previous studies found increased peak knee internal rotation post-fatigue $[5,31,40,47]$ but to date, no study reported increased knee internal rotation at IC during an SLH. Nevertheless, an extended knee position at IC during landing has been associated with increased ACL loading especially during single-leg landing [25]. Additionally, increased internal rotation of the tibia $[13,29]$ and increased $\mathrm{VGRF}_{\text {peak }}$ [7] have been associated with increased ACL loading. In summary, SAFT ${ }^{5}$ induced kinematic changes in landing strategies of an SLH which are thought to be associated with increased ACL injury risk. This evidence is in support of the notion that biomechanical screening after a functional exercise protocol such as SAFT $^{5}$ may be better suited to identify at-risk individuals than observations without prior high-intensity functional exercises. This was evident in an SLH and not in a bilateral DVJ.

Several limitations to the present study need to be acknowledged. Firstly, due to short-term characteristics of SAFT ${ }^{5}$, different physiological processes will be triggered compared to long-duration match play. More specifically, high-intensity exercises (5 min) induce accumulation of metabolites and ions resulting in metabolic acidosis [48], whereas long-duration exercises impose a greater aerobic demand and may deplete energy supply and cause dehydration [24]. This results in different loading on the body. Secondly, as training status was reported to influence fatigue-induced mechanisms [4], findings of the present study investigating recreationally active athletes cannot be generalised to elite athletes. Athletes with a higher percentage of type-1 fibres (i.e., slow/fatigue resistant fibres) and a higher aerobic fitness are suggested to have, for instance, a greater capability of resisting fatigue [4]. Finally, whilst our study involved a closer simulation of match play than maximal exhaustion protocols may do, its findings do not directly pertain to the effects of actual match play.

The present study was designed to determine the effect of SAFT on muscular and biomechanical markers of ACL injury risk, primarily to inform the development of future prospective studies that aim at including screening after functional exercises. SAFT ${ }^{5}$ reduced $\mathrm{H}_{\mathrm{ecc}}$ and $\mathrm{H}_{\mathrm{ecc}} / \mathrm{Q}_{\mathrm{con}}$ and caused altered landing patterns in SLH. All of the above have previously been associated with increased ACL injury risk and suggest that testing after a highintensity functional exercise protocol such as $\mathrm{SAFT}^{5}$ may strengthen the identification of individuals with increased ACL injury risk within a sporting population.
Conflict of interest: The authors have no conflict of interest to declare.

\section{References}

1 Bates NA, Ford KR, Myer GD, Hewett TE. Timing differences in the generation of ground reaction forces between the initial and secondary landing phases of the drop vertical jump. Clin Biomech (Bristol, Avon) 2013; 28: 796-799

2 Behrens M, Mau-Moeller A, Wassermann F, Bruhn S. Effect of fatigue on hamstring reflex responses and posterior-anterior tibial translation in men and women. PLoS One 2013; 8: e56988

3 Bishop DJ. Fatigue during intermittent-sprint exercise. Clin Exp Pharmacol Physiol 2012; 39: 836-841

4 Bogdanis GC. Effects of physical activity and inactivity on muscle fatigue. Front Physiol 2012; 3: 1-15

5 Borotikar BS, Newcomer R, Koppes R, McLean SG. Combined effects of fatigue and decision making on female lower limb landing postures: central and peripheral contributions to ACL injury risk. Clin Biomech 2008; 23: 81-92

6 Brophy R, Silvers HJ, Gonzales T, Mandelbaum BR. Gender influences: the role of leg dominance in ACL injury among soccer players. $\mathrm{Br} J$ Sports Med 2010; 44: 694-897

7 Cerulli G, Benoit DL, Lamontagne M, Caraffa A, Liti A. In vivo anterior cruciate ligament strain behaviour during a rapid deceleration movement: case report. Knee Surgery, Sport Traumatol Arthrosc 2003; 11: 307-311

8 Chappell JD, Herman DC, Knight BS, Kirkendall DT, Garrett WE, Yu B. Effect of fatigue on knee kinetics and kinematics in stop-jump tasks. Am J Sports Med 2005; 33: 1022-1029

9 Chavez A, Knudson D, Harter ROD. Activity-specific effects of fatigue protocols may influence landing kinematics: A pilot study. Int J Exerc Sci 2013; 6: 242-249

10 Cortes N, Greska E, Kollock R, Ambegaonkar J, Onate JA. Changes in lower extremity biomechanics due to a short-term fatigue protocol. J Athl Train 2013; 48: 306-313

11 Cortes N, Quammen D, Lucci S, Greska E, Onate J. A functional agility short-term fatigue protocol changes lower extremity mechanics. J Sports Sci 2012; 30: 797-805

12 Delextrat A, Gregory J, Cohen D. The use of the functional H:Q ratio to assess fatigue in soccer. Int J Sports Med 2010; 31: 192-197

13 Fleming BC, Renstrom PA, Beynnon BD, Engstrom B, Peura GD, Badger GJ, Johnson RJ. The effect of weightbearing and external loading on anterior cruciate ligament strain. J Biomech 2001; 34: 163-170

14 Garrett WE, Califf JC, Bassett FH. Histochemical correlates of hamstring injuries. Am J Sports Med 1984; 12: 98-103

15 Gehring D, Melnyk M, Gollhofer A. Gender and fatigue have influence on knee joint control strategies during landing. Clin Biomech (Bristol, Avon) 2009; 24: 82-87

16 Greco CC, Da Silva WL, Camarda SRA, Denadai BS. Fatigue and rapid hamstring/quadriceps force capacity in professional soccer players. Clin Physiol Funct Imaging 2013; 33: 18-23

17 Greig $M$. The influence of soccer-specific fatigue on peak isokinetic torque production of the knee flexors and extensors. Am J Sports Med 2008; 36: 1403-1409

18 Harriss DJ, Atkinson G. Ethical standards in sports and exercise science research: 2014 update. Int J Sports Med 2013; 34: 1025-1028

19 Hashemi J, Breighner R, Chandrashekar N, Hardy DM, Chaudhari AM, Shultz SJ, Slauterbeck JR, Beynnon BD. Hip extension, knee flexion paradox: a new mechanism for non-contact ACL injury. J Biomech 2011; 44: 577-585

20 Hawkins RD, Hulse MA, Wilkinson C, Hodson A, Gibson M. The association football medical research programme: an audit of injuries in professional football. Br J Sports Med 2001; 35: 43-47

21 Hewett TE, Myer GD, Ford KR, Heidt RS, Colosimo AJ, McLean SG, van den Bogert AJ, Paterno MV, Succop P. Biomechanical measures of neuromuscular control and valgus loading of the knee predict anterior cruciate ligament injury risk in female athletes: a prospective study. Am J Sports Med 2005; 33: 492-501

22 Hodgson M, Docherty D, Robbins D. Post-activation potentiation. Sports Med 2005; 35: 585-596

23 Hughes G, Watkins J. A risk-factor model for anterior cruciate ligament injury. Sports Med 2006; 36: 411-428

24 Krustrup P, Mohr M, Ellingsgaard H, Bangsbo J. Physical demands during an elite female soccer game: importance of training status. Med Sci Sport Exerc 2005; 37: 1242-1248 
25 Laughlin WA, WeinhandlJT, Kernozek TW, Cobb SC, Keenan KG, O'Connor $K M$. The effects of single-leg landing technique on ACL loading. J Biomech 2011; 44: 1845-1851

26 Lovell R, Knapper B, Small K. Physiological responses to SAFT90: a new soccer-specific match simulation. Coach Sport Sci 2008; 3: 46-76

27 Lucci S, Cortes N, Van Lunen B, Ringleb S, Onate J. Knee and hip sagittal and transverse plane changes after two fatigue protocols. J Sci Med Sport 2011; 14: 453-459

28 Malfait B, Sankey S, Firhad Raja Azidin RM, Deschamps K, Vanrenterghem J, Robinson MA, Staes $F$, Verschueren $S$. How reliable are lower-limb kinematics and kinetics during a drop vertical jump? Med Sci Sports Exerc 2014; 46: 678-685

29 Markolf KL, Burchfield DM, Shapiro MM, Shepard MF, Finerman GA, Slauterbeck JL. Combined knee loading states that generate high anterior cruciate ligament forces. J Orthop Res 1995; 13: 930-935

30 Marshall PWM, Lovell R, Jeppesen GK, Andersen K, Siegler JC. Hamstring muscle fatigue and central motor output during a simulated soccer match. PLoS One 2014; 9: e102753

31 McLean SG, Fellin RE, Suedekum N, Calabrese G, Passerallo A, Joy S. Impact of fatigue on gender-based high-risk landing strategies. Med Sci Sports Exerc 2007; 39: 502-514

32 Mohr M, Krustrup P, Bangsbo J. Fatigue in soccer: A brief review. J Sports Sci 2007; 23: 37-41

33 Oberländer KD, Brüggemann G-P, Höher J, Karamanidis K. Reduced knee joint moment in ACL deficient patients at a cost of dynamic stability during landing. J Biomech 2012; 45: 1387-1392

34 Oh YK, Lipps DB, Ashton-Miller JA, Wojtys EM. What strains the anterior cruciate ligament during a pivot landing? Am J Sports Med 2012; 40: $574-583$

35 Rahnama N, Reilly T, Lees A, Graham-Smith P. Muscle fatigue induced by exercise simulating the work rate of competitive soccer. J Sports Sci 2003; 21: 933-942

36 Raja Azidin RMF, Sankey S, Drust B, Robinson MA, Vanrenterghem J. Effects of treadmill versus overground soccer match simulations on biomechanical markers of anterior cruciate ligament injury risk in side cutting. J Sports Sci 2015; 1-10

37 Rampinini E, Bosio A, Ferraresi I, Petruolo A, Morelli A, Sassi A. Matchrelated fatigue in soccer players. Med Sci Sports Exerc 2011; 43: 2161-2170

38 Ruedl G, Webhofer M, Helle K, Strobl M, Schranz A, Fink C, Gatterer H, Burtscher $M$. Leg dominance is a risk factor for noncontact anterior cruciate ligament injuries in female recreational skiers. Am J Sports Med 2012; 40: 1269-1273

39 Samuelsson K, Desai N, McNair E, van Eck CF, Petzold M, Fu FH, Bhandari $M$, Karlsson J. Level of evidence in anterior cruciate ligament reconstruction research: a systematic review. Am J Sports Med 2013; 41: 924-934
40 Sanna G, O'Connor KM. Fatigue-related changes in stance leg mechanics during sidestep cutting maneuvers. Clin Biomech 2008; 23: 946-954

41 Shultz SJ, Schmitz RJ, Benjaminse A, Chaudhari AM, Collins M, Padua DA. ACL Research Retreat VI: an update on ACL injury risk and prevention. J Athl Train 2012; 47: 591-603

42 Shultz SJ, Schmitz RJ, Cone JR, Henson RA, Montgomery MM, Pye ML, Tritsch AJ. Changes in fatigue, multiplanar knee laxity, and landing biomechanics during intermittent exercise. J Athl Train 2015; 49: Epub ahead of print

43 Small K, McNaughton L, Greig M, Lovell R. The effects of multidirectional soccer-specific fatigue on markers of hamstring injury risk. J Sci Med Sport 2010; 13: 120-125

44 Small K, McNaughton LR, Greig M, Lohkamp M, Lovell R. Soccer fatigue, sprinting and hamstring injury risk. Int J Sports Med 2009; 30: $573-$ 578

45 Söderman $K$, Alfredson $H$, Pietilä $T$, Werner S. Risk factors for leg injuries in female soccer players: a prospective investigation during one outdoor season. Knee Surg Sports Traumatol Arthrosc 2001; 9: 313-321

46 Tobin DP, Delahunt E. The acute effect of a plyometric stimulus on jump performance in professional rugby players. J Strength Cond Res 2014; 28: 367-372

47 Tsai L-C, Sigward SM, Pollard CD, Fletcher MJ, Powers CM. Effects of fatigue and recovery on knee mechanics during side-step cutting. Med Sci Sports Exerc 2009; 41: 1952-1957

48 Tschakert G, Kroepfl J, Hofmann P, Mueller A, Moser O, Groeschl W. How to regulate the acute physiological response to "aerobic" highintensity interval exercise. J Sport Sci Med 2015; 14: 29-36

49 Vanrenterghem J, Gormley D, Robinson MA, Lees A. Solutions for representing the whole-body centre of mass in side cutting manoeuvres based on data that is typically available for lower limb kinematics. Gait Posture 2010; 31: 517-521

50 Vøllestad NK. Measurement of human muscle fatigue. J Neurosci Methods 1997; 74: 219-227

51 Wikstrom EA, Powers ME, Tillman MD. Dynamic stabilization time after isokinetic and functional fatigue. J Athl Train 2004; 39: 247-253

52 Yeung SS, Suen AMY, Yeung EW. A prospective cohort study of hamstring injuries in competitive sprinters: preseason muscle imbalance as a possible risk factor. Br J Sports Med 2009; 43: 589-594

53 Yu B, Queen RM, Abbey AN, Liu Y, Moorman CT, Garrett WE. Hamstring muscle kinematics and activation during overground sprinting. J Biomech 2008; 41: 3121-3126

54 Zebis MK, Bencke J, Andersen LL, Alkjaer T, Suetta C, Mortensen P, Kjaer $M$, Aagaard $P$. Acute fatigue impairs neuromuscular activity of anterior cruciate ligament-agonist muscles in female team handball players. Scand J Med Sci Sports 2011; 21: 833-840 


\section{Appendix}

$\nabla$
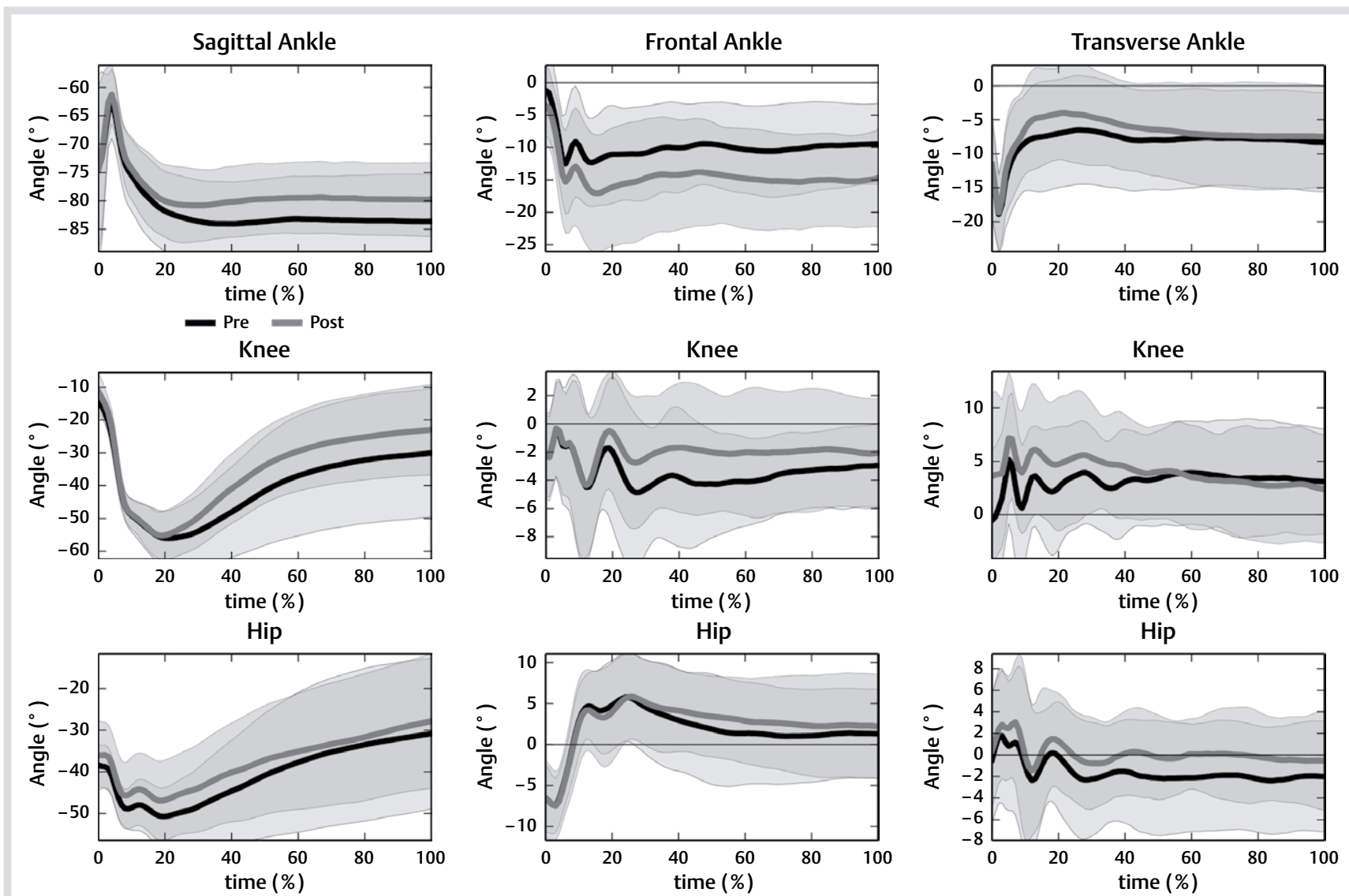

Appendix 1 Ankle, knee and hip angles $\left({ }^{\circ}\right)(m e a n \pm S D$ ) of sagittal, frontal and transversal plane during stance phase (initial contact $+1 \mathrm{~s}$ ) of a single-leg hop pre and immediately post SAFT5. Black solid line represents PRE and red solid line represents POST. 

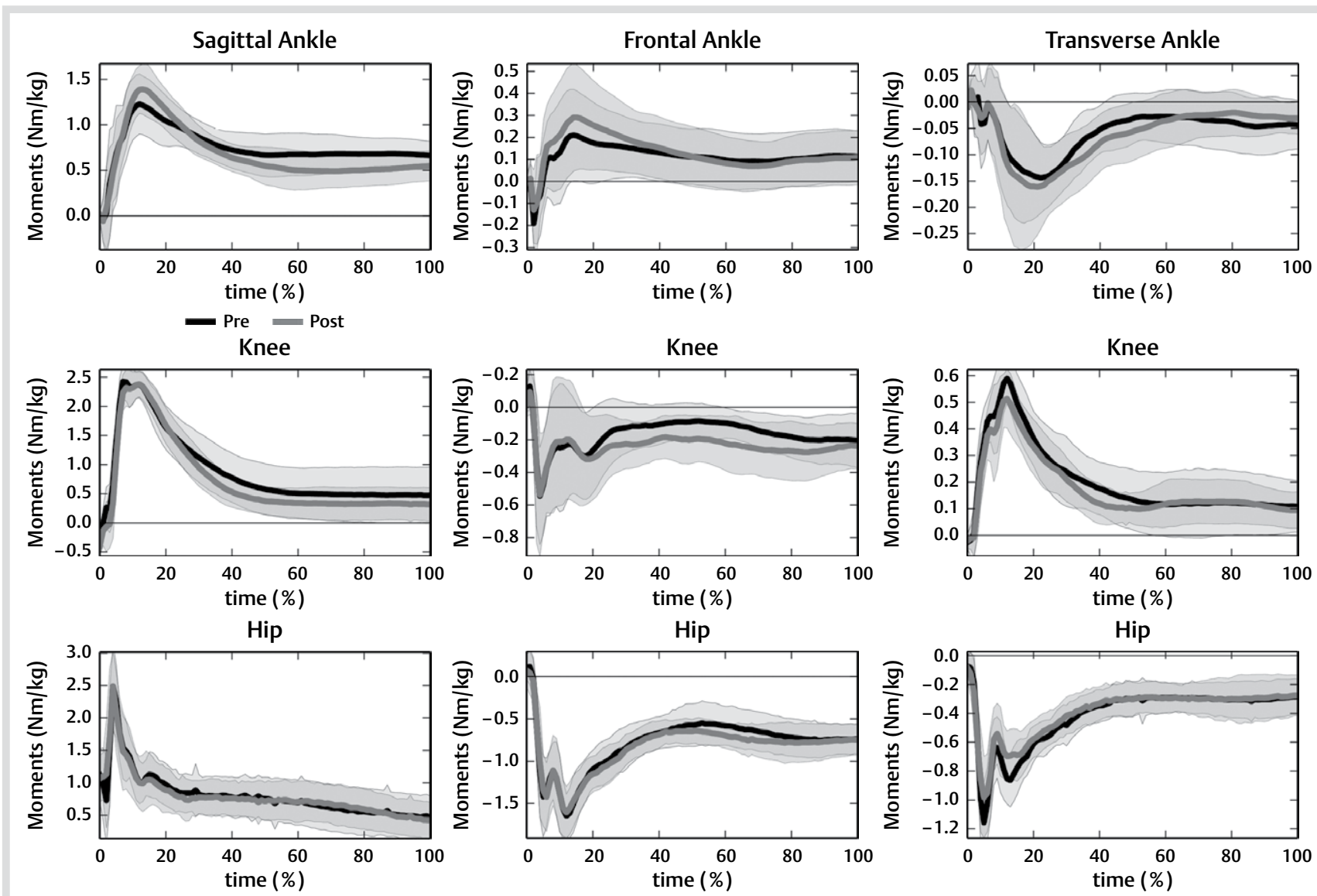

Appendix 2 Ankle, knee and hip moments $\left(\mathrm{Nm}_{\mathrm{kg}}{ }^{-1}\right)$ (Mean $\pm \mathrm{SD}$ ) of the sagittal, frontal and transversal plane during the stance phase (initial contact $+1 \mathrm{~s}$ ) of a single-leg hop pre and immediately post SAFT ${ }^{5}$. Black solid line represents PRE and red solid line represents POST. 